\title{
Fractional Flow Reserve
}

\author{
Wilbert S. Aronow, MD, FACC, FAHA \\ Department of Medicine, Division of Cardiology, Westchester Medical Center/New York Medical \\ College, Valhalla, NY, USA \\ wsaronow@aol.com
}

\begin{abstract}
Traditionally, coronary artery disease was assessed using coronary angiographic guidance. Significant lesions were determined by the coronary angiographer. However, interobserver variability showed a significant limitation of interpretation of coronary angiography. Stent deployment and apposition were also evaluated based on angiographic appearance only. Risk factors for stent thrombosis and in-stent restenosis such as incomplete stent apposition, edge dissection, and thrombus presence were often not detected. However, in the last 10 years, the field of interventional cardiology has benefitted from the use of more objective measures of the severity of coronary artery disease. Fractional flow reserve is calculated by dividing the distal coronary pressure by the proximal coronary pressure during maximal hyperemia this article will discuss the use of fractional flow reserve in determining the severity of coronary artery lesions and whether they should be revascularized.
\end{abstract}

Keywords: coronary artery disease; fractional flow reserve percutaneous coronary intervention

Coronary artery disease is the leading cause of mortality internationally. Traditionally, coronary artery disease was assessed using coronary angiographic guidance. Significant lesions were determined by the coronary angiographer. However, interobserver variability showed a significant limitation of interpretation of coronary angiography [1]. Stent deployment and apposition were also evaluated based on angiographic appearance only. Risk factors for stent thrombosis and in-stent restenosis such as incomplete stent apposition, edge dissection, and thrombus presence were often not detected [2]. However, in the last 10 years, the field of interventional cardiology has benefitted from the use of more objective measures of the severity of coronary artery disease. This article will discuss the use of fractional flow reserve in determining the severity of coronary artery lesions and whether they should be revascularized.

\section{FraCtional FLOW RESERVE}

Over the last 10 years, fractional flow reserve has become a reference standard for the invasive assessment of coronary artery disease. Its measurement assesses the fuctional severity of coronary artery stenoses. and the need for coronary revascularization [3, 4]. Fractional flow reserve is calculated by dividing the distal coronary pressure by the proximal coronary pressure during maximal hyperemia [3]. In an ideal situation with no limitation to coronary flow, these pressures should be equal. The hemodynamic significance of a coronary lesion is determined by the ratio of these pressures. Pijils et al demonstrated in follow-up of patients in the Deferral of Percutaneous Coronary Intervention (DEFER) study that a fractional flow reserve cutoff of 0.75 was excellent in predicting 5year outcome after deferral of percutaneous coronary intervention of an intermediate coronary stenosis [5]. This study found that percutaneous coronary intervention of a functionally nonsignificant stenosis indicated by a fractional flow reserve of $\geq 0,75$ is not of benefit to the patient [5]. This study also demonstrated that the coronary lesions at greatest risk of causing cardiovascular death or myocardial infarction are those that are functionally significant as indicated by a fractional flow reserve of $<0.75[5]$.

The Fractional Flow Reserve Versus Angiography for Guiding Percutaneous Coronary Intervention (FAME) trials further solidified the role of fractional flow reserve in the treatment of patients with multi-vessel coronary artery disease [6-9]. In the FAME 1 trial, fractional flow reserve - guided percutaneous coronary intervention with drug- eluting stents was demonstrated to have a lower composite of death, myocardial infarction, and repeat revascularization as compared to interventions done based on an angiographic assessment alone [6]. The 1-year rate of death, nonfatal myocardial infarction, and repeat revascularization in the 1005 patients with multivessel coronary artery disease 
randomized to percutaneous coronary intervention with drug-eluting stents guided by coronary angiography alone or to fractional flow reserve measurements plus coronary angiography was $18.3 \%$ (91 patients) in the coronary angiography alone group versus $13.2 \%$ (67 patients) in the fractional flow reserve group $(\mathrm{p}=0.02)$ [6]. At 1 year follow-up $78 \%$ of the coronary angiography alone group and $81 \%$ of the fractional flow reserve group were free from angina pectoris $(p=0.20)$ [6]. The number of stents used was 2.7 in the fractional flow reserve group versus 1.9 in the coronary angiography group alone $(\mathrm{p}<0001)[6]$

At 2-year follow-up of FAME 1, the rates of mortality or myocardial infarction were $12.9 \%$ in the angiography-guided group versus $8.4 \%$ in the fractional flow reserve group $(p=0.02)$ [7]. The 2-year rates of death, nonfatal myocardial infarction, or coronary revascularization were $22.4 \%$ in the angiography-guided group versus $17.9 \% \%$ in the fractional flow reserve group $(p=0.08)$ [7]. The 2year rates of percutaneous coronary intervention or coronary artery bypass graft surgery were $12.7 \%$ in the angiography-guided group versus $10.6 \% \%$ in the fractional flow reserve group $(p=0.30)$ [7].For coronary lesions deferred on the basis of a fractional flow reserve greater than 0.80 , the 2-year incidence of myocardial infarction was $0.2 \%$, and the 2 -year incidence of coronary revascularization was $3.2 \%$ [7]

At 5-year follow-up of FAME 1, the incidence of major adverse cardiac events was $31 \%$ in the angiography-guided group versus $28 \% \%$ in the fractional flow reserve group $(p=0.31)$ [8]. The number of stents used was 2.7 in the fractional flow reserve group versus 1.9 in the coronary angiography group alone $(\mathrm{p}<0001)[8]$

The utility of fractional flow reserve was again shown in the FAME 2 trial, which demonstrated that percutaneous coronary intervention in addition to maximal medical therapy was superior to maximal medical therapy alone in 888 randomized patients with hemodynamically significant lesions as demonstrated by a fractional flow reserve value of less than 0.80 . [9]. The primary endpoint of death, myocardial infarction, or urgent coronary revascularization was $4.3 \%$ in the percutaneous coronary intervention group versus $12.7 \%$ in the maximal medical therapy alone group (hazard ratio $=0.32, p$ <0.001) [9]. This was driven primarily by a reduction in the amount of urgent coronary revascularization in the percutaneous coronary intervention group (1.6\%) than in the maximal medical therapy alone group $(11.1 \%$ ) (Hazard ratio $=0.13, \mathrm{p}<0.001$ ) [9]. In patients without myocardial ischemia, the outcome was favorable with maximal medical therapy alone [9].

The implications of these findings are of importance. The Clinical Outcomes Utilizing Revascularization and Aggressive Drug Evaluation (COURAGE) trial demonstrated that percutaneous coronary intervention offered no mortality benefit in patients with stable coronary artery disease as compared to optimal medical therapy [10]. However, in patients with significant myocardial ischemia, there may be a decrease in major cardiovascular events with percutaneous coronary intervention. Therefore, fractional flow reserve when used as a surrogate marker for ischemia, allows for a direct intervention to an ischemia- causing lesion, which may cause a reduction in major cardiovascular events. Fractional flow reserve assessment of coronary artery disease can also cause deferral of revascularization of lesions that are not hemodynamically significant. The International Study of Comparative Health Effectiveness with Medical and Invasive Approaches (ISCHEMIA) trial is enrolling stable ischemic heart disease patients with at least moderate ischemia who are asymptomatic or symptomatic with or without previous revascularization to investigate whether an invasive strategy with revascularization will improve prognosis compared with optimal medical therapy 11].

Sant'Anna et al demonstrated that fractional flow reserve assessment in multi-vessel coronary artery disease has shown a decrease in the number of significant lesions [12]. In this study of 250 patients with 471 coronary stenoses scheduled for percutaneous coronary intervention, $32 \%$ of the coronary stenoses and $48 \%$ of the patients would have received a different treatment if a fractional flow reserve measurement was not made [12]. Other studies have similarly found that in patients with multi-vessel coronary artery disease, fractional flow reserve - guided percutaneous coronary intervention leads to less arteries being treated than angiographically treated vessels [13].

Clinical trials have mostly suggested that the risk of major adverse cardiovascular outcomes after fractional flow reserve based deferral of percutaneous coronary intervention is low. However, studies have been variable with respect to the rate of future intervention on these vessels, with the majority suggesting a rate of less than $10 \%$ at one year. Depta et al recently showed that deferral of 
percutaneous coronary intervention based on fractional flow reserve resulted in delayed intervention of 5.3\% at 1 year and 18\% within 4 years [14]. A clinical prediction model including age, history of tobacco use, history of coronary artery disease, higher creatinine, and multivessel coronary artery disease plus the fractional flow reserve value can help predict the risk of deferred lesion intervention in the first year after fractional flow reserve assessment [14].

Left main coronary artery stenosis is also an important dilemma. Angiographically, the severity of left main coronary artery lesions can vary based on the view selected. A hemodynamic assessment of the left main coronary artery stenosis may help with this ambiguity in light of the potential clinical implications. Courtis et al showed that an fractional flow reserve - guided assessment of left main coronary artery stenosis resulted in similar outcomes in patients treated with revascularization versus medical therapy [15]. This study investigated 142 patients with ambiguous or intermediate left main coronary artery lesions and decided on coronary revascularization based on whether the fractional flow reserve was less than 0.75 or greater than 0.80 . Those coronary artery lesions which were less than 0.75 were treated with coronary revascularization therapy (60 patients) and those with fractional flow reserve values greater than 0.80 were treated with medical therapy ( 82 patients). At 14-months follow-up, the incidence of major adverse cardiac events was $13 \%$ in the medical therapy group versus $7 \%$ with coronary revascularization $(p=0.27)[15]$

Perhaps one of the most clinically important applications of these methods is the assessment of the degree of left main coronary artery disease. Traditionally, visual assessment of left main coronary artery disease has been variable and can be clinically important. Diffuseness of the atherosclerotic process seems to be the major reason for angiographic underestimation of narrowing of coronary arteries [16].

Bech et al measured fractional flow reserve in 54 consecutive patients with angiographically equivalent left main coronary artery disease[17]. The fractional flow reserve was $\geq 0.75$ in 24 of 54 patients $(44 \%)$ who were treated medically. Coronary artery bypass graft surgery was performed in the 30 of 54 patients $(56 \%)$ who had a fractional flow reserve less than 0.75 [17]. Survival at 3 years was $100 \%$ for the medical group and $97 \%$ for the surgical group [17]. Event-free survival at 3 years was $76 \%$ for the medical group and $83 \%$ for the surgical group [17].

In 213 patients with an angiographically equivocal left main coronary artery stenosis, quantitative coronary angiography and measurements of fractional flow reserve were performed [18]. The fractional flow reserve was less than 0.80 in 75 patients and $\geq 0.80$ in 138 patients. When the fractional flow reserve was less than 0.80 , coronary artery bypass surgery was performed. When the fractional flow reserve was $\geq 0.80$, the patients were treated medically or another coronary artery stenosis was treated by coronary angioplasty (the nonsurgical group). The 5-year survival estimates were $85.4 \%$ in the surgical group versus $89.8 \%$ in the nonsurgical group (p not significant). The 5year event-free survival estimates were $82.8 \%$ in the surgical group versus $74.2 \%$ in the nonsurgical group ( $\mathrm{p}$ not significant). Percent diameter stenosis measured by quantitative coronary angiography correlated with fractional flow reserve ( $\mathrm{p}<0.001)$, but a very large scatter was present. In $23 \%$ of patients with a diameter left main coronary artery stenosis, the left main coronary artery stenosis was hemodynamically significant by measurement of fractional flow reserve [18]. These data suggest that fractional flow reserve should be measured in patients with equivocal stenosis of the left main coronary artery before making a decision about the need for coronary revascularization [18].

There is still some controversy as to which fractional flow reserve value should be used as the value for coronary revascularization and how distal left main coronary artery stenosis should be approached. Perhaps, intravascular ultrasound is most useful in cases where borderline fractional flow reserve readings are attained. Ostial and mid-shaft left main coronary artery disease may be able to be reliably assessed with fractional flow reserve, but distal left main coronary artery disease can be more cumbersome as the disease often extends into the daughter vessels. Some coronary interventionalists recommend a pressure wire pullback method from the daughter vessels into the left main coronary artery in order to localize the most significant disease [19]. 


\section{REFERENCES}

[1] Zir LM, Miller SW, Dinsmore RE, et al. Interobserver variability in coronary angiography. Circulation. 1976; 53:627-632.

[2] Reejhsinghani R, Lotfi AS. Prevention of stent thrombosis: challenges and solutions. Vasc Health Risk Manag. 2015; 11:93-106.

[3] Pijls NHJ, de Bruyne B, Peels K, et al. Measurement of fractional flow reserve to assess the functional severity of coronary-artery stenoses. N Engl J Med. 1996;334:1703-1708.

[4] Pijls NHJ. Fractional flow reserve to guide coronary revascularization. Circ J. 2013; 77:561-569.

[5] Pijls NHJ, van Schaardenburgh P, Manoharan G, et al. Percutaneous coronary intervention of functionally nonsignificant stenosis: 5-year follow-up of the DEFER Study. J Am Coll Cardiol. 2007; 49:2105-2111.

[6] Tonino PAL, De Bruyne B, Pijls NHJ, et al. Fractional flow reserve versus angiography for guiding percutaneous coronary intervention. N Engl J Med. 2009; 360:213-224.

[7] Piljs NH, Fearon WF, Tonino PA, et al. Fractional flow reserve versus angiography for guiding percutaneous coronary intervention in patients with multivessel coronary artery disease: 2-year follow-up of the FAME (Fractional Flow Reserve Versus Angiography for Multivessel Evaluation) mstudy. J Am Coll Cardiol 2010; 56: 177-184.

[8] Van Nunen LX, Zimmermann FM, Tonino PAL, et al. Fractional flow reserve versus angiography for guidance of PCI in patients with multivessel coronary artery disease (FAME): 5year follow-up of a randomized controlled trial. Lancet 201`5; 386: 1853-1860.

[9] De Bruyne B, Pijls NHJ, Kalesan B, et al. Fractional flow reserve-guided PCI versus medical therapy in stable coronary disease. N Engl J Med. 2012; 367:991-1001.

[10] Boden WE, O'Rourke RA, Teo KK, et al. Optimal medical therapy with or without PCI for stable coronary disease. N Engl J Med 2007; 356: 1503-1516.

[11] Maron DJ, Hochman JS. Revascularization for silent ischemia? : another piece of the puzzle. J Am Coll Cardiol 2013; 61: 1624-1625.

[12] Sant'Anna FM, Silva EER, Batista LA,et al. Influence of routine assessment of fractional flow reserve on decision making during coronary interventions. Am J Cardiol. 2007; 99:504-508.

[13] Wongpraparut N, Yalamanchili V, Pasnoori V, et al. Thirty-month outcome after fractional flow reserve-guided versus conventional multivessel percutaneous coronary intervention. Am J Cardiol. 2005; 96:877-884.

[14] Depta JP, Patel JS, Novak E, Gage BF, et al. Risk model for estimating the 1-year risk of deferred lesion intervention following deferred revascularization after fractional flow reserve assessment. Eur Heart J. 2015 Feb 21; 36:509-15.

[15] Courtis J, Rodes-Cabau J, Larose E, et al. Usefulness of coronary fractional flow reserve measurements in guiding clinical decisions in intermediate or equivocal left main coronary stenoses. Am J Cardiol. 2009; 103:943-949.

[16] . Arnett EN, Isner JM, Redwood DR, et al. Coronary artery narrowing in coronary heart disease: comparison of cineangiographic and necropsy findings. Ann Intern Med. 1979; 91:350-356.

[17] Bech GJ, Droste H, Pijls NH, et al. Value of fractional flow reserve in making decisions about bypass surgery for equivocal left main coronary artery disease. Heart. 2001; 86:547-552.

[18] Hamilos M, Muller O, Cuisset T, et al. Long-term clinical outcome after fractional flow reserveguided treatment in patients with angiographically equivocal left main coronary artery stenosis. Circulation. 2009; 120:1505-1512.

[19] Puri R, Kapadia SR, Nicholls SJ, et al. Optimizing outcomes during left main percutaneous coronary intervention with intravascular ultrasound and fractional flow reserve: the current state of evidence. JACC Cardiovasc Interv. 2012; 5:697-707. 